\title{
Editorial
}

\section{Function Spaces, Fixed Points, Approximations, and Applications}

\author{
Giuseppe Marino, ${ }^{1,2}$ Filomena Cianciaruso, ${ }^{1}$ Nawab Hussain, ${ }^{2}$ and Enrique Llorens Fuster ${ }^{3}$ \\ ${ }^{1}$ Department of Mathematics and Computer Sciences, University of Calabria, 87036 Arcavacata, Rende, Italy \\ ${ }^{2}$ Department of Mathematics, King Abdulaziz University, Jeddah 21589, Saudi Arabia \\ ${ }^{3}$ Department of Mathematical Analysis, University of Valencia, Valencia, Spain \\ Correspondence should be addressed to Giuseppe Marino; giuseppe.marino@unical.it
}

Received 16 February 2017; Accepted 16 February 2017; Published 27 March 2017

Copyright (C) 2017 Giuseppe Marino et al. This is an open access article distributed under the Creative Commons Attribution License, which permits unrestricted use, distribution, and reproduction in any medium, provided the original work is properly cited.

An important branch of nonlinear analysis theory, applied in the study of nonlinear phenomena in engineering, physics, and life sciences, is related to the existence of fixed points of nonlinear mappings, to the approximation of fixed points of nonlinear operators and of zeros of nonlinear operators, and to the approximation of solutions of variational inequalities.

This special issue is focused on the latest achievements on these topics and the related applications.

The aim is to present newest and extended coverage of the fundamental ideas, concepts, and important results on the topics below in general function spaces useful in analysis.

The search for the solutions of equations (ordinary and partial differential equations, functional differential equations, integral equations, etc.) is currently studied in specific spaces of functions.

The choice of fixed point theorems to be applied is conditioned by the underlying functions space.

We invited the authors to present their original articles that will stimulate the continuing efforts in developing new results in the previous mentioned areas.

The selected and published papers can be entered in the following areas.

(i) Function Spaces. J. R. Acosta-Portilla et al. characterized the family of nonexpansive mappings which are invariant under renormings and they also compared the families of nonexpansive mappings under two equivalent norms.

$\mathrm{X}$. Li et al. defined and studied some subclasses of multivalent analytic functions of higher order in the unit disc. These classes generalize some classes previously studied.
They obtained coefficient inequalities, distortion theorems, extreme points, and integral mean inequalities.

W. Wang et al. considered the question, what is the appropriate formulation of Godefroy-Shapiro criterion for tuples of operators? And they introduced a new notion about tuples of operators S-mixing, which lies between mixing and weakly mixing. They also obtained a sufficient condition to ensure a tuple of operators to be S-mixing. Moreover, they studied some new properties of S-mixing operators on several concrete Banach spaces.

(ii) Fixed Points. J. Tiammee et al. proved some fixed points theorems for multivalued nonself $G$-almost contractions in Banach spaces with a directed graph and given some examples to illustrate the main results.

B. Z. Popovic et al. established a unique fixed point theorem for three self-maps under rational type contractive condition. In addition, a unique fixed point result for six continuous self-mappings through rational type expression is also discussed.

N. Hussain et al. have highlighted that remarkable feature of contractions is associated with the concept MizoguchiTakahashi function. For the purpose of extension and modification of classical ideas related to Mizoguchi-Takahashi contraction, they defined generalized Mizoguchi-Takahashi $G$-contractions and established some generalized fixed point theorems regarding these contractions.

(iii) Approximation. I. Altun et al. provided sufficient conditions for the existence of a unique common fixed point for 
a pair of mappings $T, S$ defined on a metric space. Moreover, a numerical algorithm is presented in order to approximate such solution. Their approach is different from the usually used methods in the literature.

(iv) Fixed Point Theory Applied to Differential Problems. Y. Zhang and J. Zhu presented a new nonlinear contraction principle on partial metric spaces and proved the existence of common fixed point. They have also given some examples to apply their results to study the existence of common bounded solution of the system of functional equations.

R. Rao and S. Zhong employed Banach fixed point theorem to derive LMI-based exponential stability of impulsive Takagi-Sugeno (T-S) fuzzy integrodifferential equations, originated from Cohen-Grossberg Neural Networks.

J.-P. Sun et al. studied a system of third-order threepoint boundary value problems. By imposing some suitable conditions on the functions, they obtained the existence of at least one positive solution of the system. The main tool used is the theory of the fixed point index.

(v) Differential Problems. Q. Sun and Y. Cui investigated a $(k, n-k)$ conjugate boundary value problem with integral boundary conditions. By using Mawhin continuation theorem, they studied the solvability of this boundary value problem at resonance.

\section{Acknowledgments}

The editors would like to thank the authors for their interesting contributions

Giuseppe Marino

Filomena Cianciaruso

Nawab Hussain

Enrique Llorens Fuster 


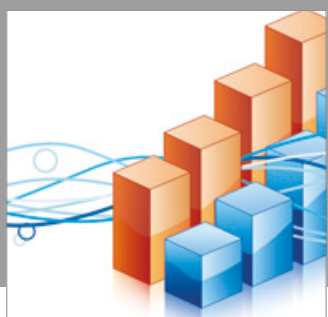

Advances in

Operations Research

vatersals

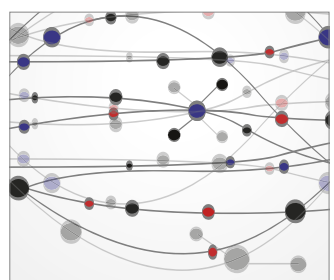

\section{The Scientific} World Journal
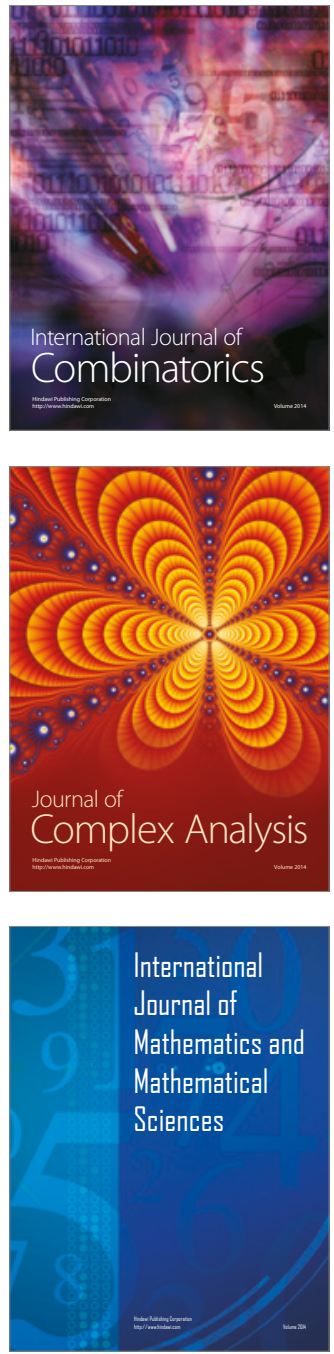
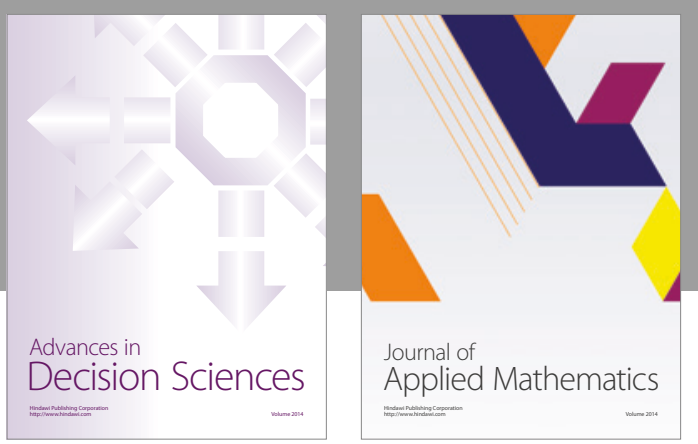

Algebra

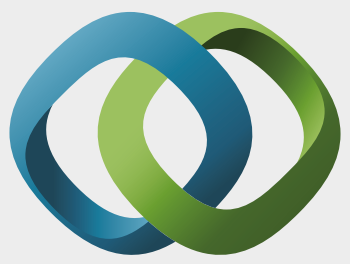

\section{Hindawi}

Submit your manuscripts at

https://www.hindawi.com
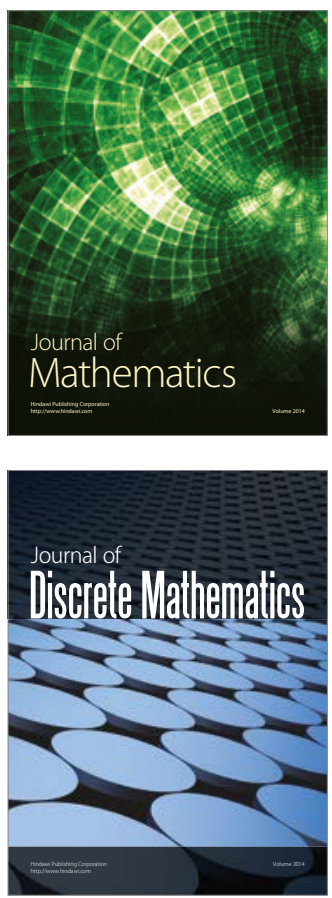

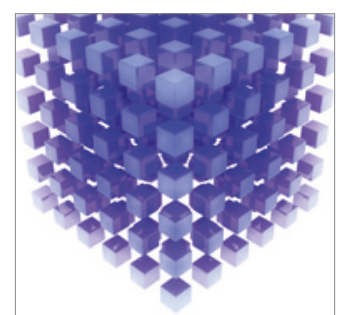

Mathematical Problems in Engineering
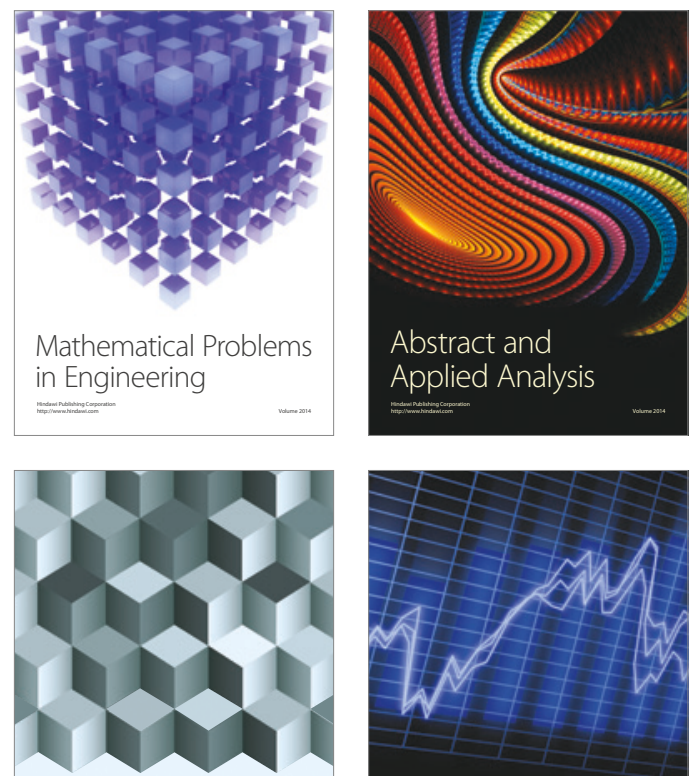

Journal of

Function Spaces

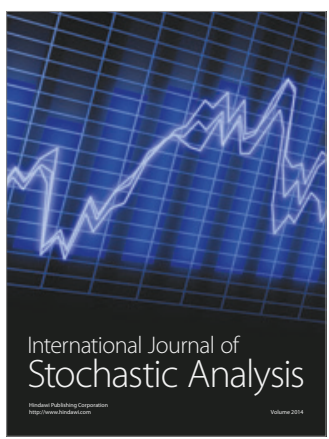

Probability and Statistics
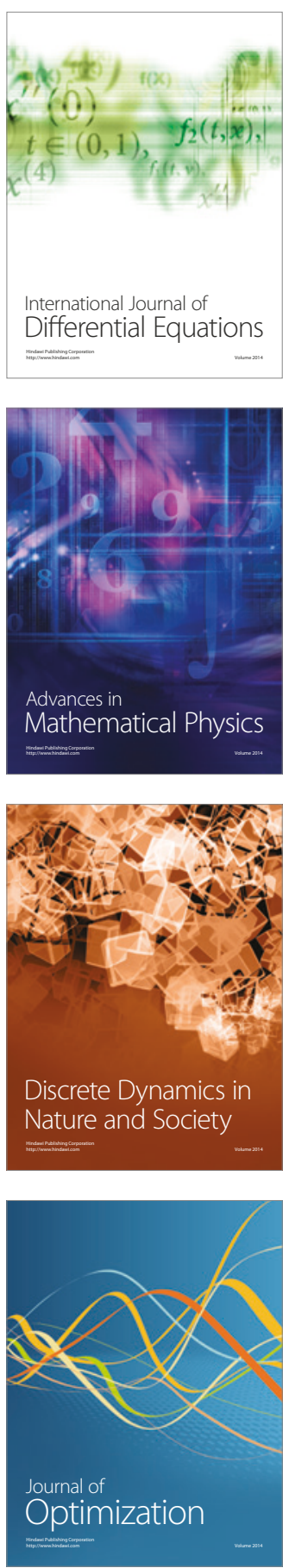